\title{
Testing BRNBU Ageing Class of Life-Time Distribution Based on Moment Inequality
}

Touseef Ahmed A 11 and Rizwan $\mathrm{U}^{*, 2}$

Received: 12 February 2021/ Accepted: 12 May 2021/ Published online: 18 June 2021

(C)Sacred Heart Research Publications 2017

\begin{abstract}
In this paper, new moment inequality is derived for Bivariate Renewal New Better than Used (BRNBU) ageing class of life-time distribution. This inequality demonstrates that if the mean life is finite, then all higher order moments exist. Based on the Moment inequality, new testing procedures for testing bivariate exponentiality against BRNBU ageing class of life-time distribution is introduced.The asymptotic normality of the test statistic and its consistency are studied. Using Monte Carlo Method, critical values of the proposed test are calculated for $n=5(5) 100$ and tabulated. Finally, the theoretical results are applied to analyze real-life data sets.
\end{abstract}

Key words: Classes of Life-Time Distribution, BRNBU, Moment Inequality, U-Statistic, Life Testing .

AMS classification: $60 \mathrm{~K} 10$

\section{Introduction}

Certain classes of life-time distributions and their variations have been introduced in reliability theory, the applications of these classes of life-time distributions can be seen in engineering, biological science, maintenance and biometrics. The main aim of constructing new tests is to gain higher efficiencies.

Bivariate ageing classes of life-time distributions have been found to be useful in reliability analysis, applications in engineering, queueing theory, medical diagonotics and many other fields. There are many situations where a continuous time is inappropriate for describing the lifetime of devices and other systems. For example, the life time of many devices in industry such as switches and mechanical tools, depends essentially on the number of times they are turned on and off or the number

\footnotetext{
${ }^{1,2}$ Department of Mathematics, Islamiah College(Autonomous), Vaniyambadi - 635 752, Tirupattur Dt, Tamilnadu, India. Email: ${ }^{1}$ touseefmath1@gmail.com, ${ }^{*, 2}$ rizwanphd9@gmail.com
} 
of shocks they receive. In such cases, the time to failure is often more appropriately represented by the number of times they are used before they fail, which is a discrete random variable. Bivariate distributions are observed in different practical situations, for example, in the survival of the paired organs such as persons eyes,ears, kidneys and lungs, or in the survival of two-engine airplane.

Testing bivariate exponentiality against some bivariate ageing classes of life-time distributions has seen a good deal of attention. The moment inequality for the Bivariate Renewal New Better than Used (BRNBU) Ageing Class of life-time distribution can be found in the work of [6]. Now we propose a test statistic testing Bivariate Exponentiality Against Bivariate Renewal New Better than Used (BRNBU) Ageing Class of life-time distribution, based on the moment inequality.

The rest of the paper is arranged as follows; In section 2, the preliminaries required for the further discussion is given. In section 3, moment inequality for the Bivariate Renewal New Better than Used (BRNBU) Ageing Class of life-time distribution is derived. A new test statistic for Bivariate Renewal New Better than Used (BRNBU) Ageing Class of life-time distribution based on moment inequality is proposed in section 4. Using Monte Carlo Method critical values of the proposed test statistic are calculated for $n=5(5) 100$ and tabulated in section 5. The application of the proposed test to real data sets is discussed in section 6 . Finally, conclusion is given in section 7 .

\section{Preliminaries}

Let $(X, Y)$ denote the survival time of a device having a joint distribution function $F(x, y)$. The bivariate joint survival function is given by

$$
\bar{F}(x, y)=P(X>x, Y>y), \quad x, y \geq 0
$$

where it is assumed that $\bar{F}(0,0)=1$.

The following definition of Bivariate ageing classes of life-time distributions appeared in [6].

Definition 2.1 A bivariate random variable $(X, Y)$ or its distribution $\bar{F}(x, y)$ is said to have Bivariate Renewal New Better than Used (BRNBU) ageing class, if

$$
\begin{aligned}
& \bar{W}(x+t, y+s) \leq \bar{W}(x, y) \bar{W}(t, s), \text { for all } x, y, t, s \geq 0, \text { where } \\
& \bar{W}(t, s)=\frac{1}{\mu} \int_{t}^{\infty} \int_{s}^{\infty} \bar{F}(u, v) d v d u,
\end{aligned}
$$

$\mu=\int_{0}^{\infty} \int_{0}^{\infty} \bar{F}(x, y) d y d x$ denotes the mean of $F$ and is assumed to be finite. 
ISSN: 2456-8686, 5(1), 2021:001-010

https://doi.org/10.26524/cm87

Definition 2.2 The $r$-th Moment of a bivariate random variable $(X, Y)$ is

$$
\mu_{r}=E\left(X^{r} Y^{r}\right)=r^{2} \int_{0}^{\infty} \int_{0}^{\infty}(x y)^{r-1} \bar{F}(x, y) d y d x .
$$

\section{Moment Inequality}

A moment inequality is derived for the system whose life-time distribution is a Bivariate Renewal New Better than Used ageing class of life-time distribution.

Theorem 3.1 If $F$ is a Bivariate Renewal New Better than Used ageing class of life-time distribution, then moments of all order $r$, for all integer $r \geq 0$, exist and are finite. Here $\mu$ denotes the mean of $F$.

$$
\frac{\mu_{r+3}}{(r+2)(r+3)} \leq \frac{1}{\mu}\left[\sum_{i=0}^{r}\left(\begin{array}{l}
r \\
i
\end{array}\right) \frac{1}{(r-i+1)(i+1)} \frac{\mu_{r-i+2} \mu_{i+2}}{(r-i+2)(i+2)}\right] \text {. }
$$

Proof: Since $F$ is a Bivariate Renewal New Better than Used ageing class of life-time distribution, we have

$$
\bar{W}(x+t, y+s) \leq \bar{W}(x, y) \bar{W}(t, s)
$$

for all $x, y, t, s \geq 0$, where

$$
\bar{W}(t, s) \frac{1}{\mu} \int_{t}^{\infty} \int_{s}^{\infty} \bar{F}(u, v) d v d u
$$

Multiplying both side by $(x+t)^{r}(y+s)^{r}$ and integrating, we get

$$
\begin{aligned}
\int_{0}^{\infty} \int_{0}^{\infty} \int_{0}^{\infty} \int_{0}^{\infty}(x+t)^{r}(y+s)^{r} \bar{W}(x+t, y+s) d y d x d s d t \\
\quad \leq \int_{0}^{\infty} \int_{0}^{\infty} \int_{0}^{\infty} \int_{0}^{\infty}(x+t)^{r}(y+s)^{r} \bar{W}(x, y) \bar{W}(t, s) d y d x d s d t
\end{aligned}
$$

Consider,

$$
\begin{array}{r}
\int_{0}^{\infty} \int_{0}^{\infty} \int_{0}^{\infty} \int_{0}^{\infty}(x+t)^{r}(y+s)^{r} \bar{W}(x+t, y+s) d y d x d s d t \\
=\frac{1}{\mu} \int_{0}^{\infty} \int_{0}^{\infty} \bar{F}(x, y) \int_{0}^{X} \int_{0}^{Y}(u v)^{r+1} d v d u d x d y \\
=\frac{1}{\mu} E\left[\int_{0}^{\infty} \int_{0}^{\infty} \frac{(x y)^{r+2}}{r+2} I[X>x, Y>y] d x d y\right]
\end{array}
$$


ISSN: 2456-8686, 5(1), 2021:001-010

https://doi.org/10.26524/cm87

$$
\begin{aligned}
&=\frac{1}{\mu} \frac{1}{(r+2)} E(X Y)^{r+2} \\
&=\frac{\mu_{r+3}}{\mu(r+2)(r+3)}
\end{aligned}
$$

where $I$ is a indicator function. Further,

$$
\begin{aligned}
\int_{0}^{\infty} \int_{0}^{\infty} \int_{0}^{\infty} \int_{0}^{\infty}(x+t)^{r}(y+s)^{r} \bar{W}(x, y) \bar{W}(t, s) d y d x d s d t \\
=\frac{1}{\mu^{2}} \sum_{i=0}^{r}\left(\begin{array}{l}
r \\
i
\end{array}\right) \int_{0}^{\infty} \int_{0}^{\infty} \int_{0}^{\infty} \int_{0}^{\infty} x^{r-i} t^{i} y^{r-i} s^{i} \bar{W}(x, y) \bar{W}(t, s) d x d y d s d t \\
=\frac{1}{\mu^{2}} \sum_{i=0}^{r}\left(\begin{array}{l}
r \\
i
\end{array}\right)\left(\int_{0}^{\infty} \int_{0}^{\infty}(x y)^{r-i} \bar{W}(x, y) d x d y\right)\left(\int_{0}^{\infty} \int_{0}^{\infty}(t s)^{i} \bar{W}(t, s) d s d t\right) \\
=\frac{1}{\mu^{2}} \sum_{i=0}^{r}\left(\begin{array}{l}
r \\
i
\end{array}\right) \int_{0}^{\infty} \int_{0}^{\infty} \frac{(x y)^{r-i+1}}{(r-i+1)} I(X>x, Y>y) d x d y \times \\
E\left[\int_{0}^{\infty} \int_{0}^{\infty} \frac{(t s)^{i+1}}{(i+1)} I(T>t, S>s) d s d t\right] \\
=\frac{1}{\mu^{2}} \sum_{i=0}^{r}\left(\begin{array}{l}
r \\
i
\end{array}\right) \frac{1}{(r-i+1)(i+1)} \frac{\mu_{r-i+2}}{(r-i+2)} \frac{\mu_{i+2}}{(i+2)}
\end{aligned}
$$

Using the equation (2) and (3), the inequality (1) becomes

$$
\frac{\mu_{r+3}}{(r+2)(r+3)} \leq \frac{1}{\mu}\left[\sum_{i=0}^{r}\left(\begin{array}{l}
r \\
i
\end{array}\right) \frac{1}{(r-i+1)(i+1)} \frac{\mu_{r-i+2} \mu_{i+2}}{(r-i+2)(i+2)}\right] \text {. }
$$

This completes the proof of theorem.

\section{Testing Bivariate Exponentiality against Bivariate Renewal New Better than Used Ageing Class}

Using inequality (4), we test the null hypothesis

$H_{0}: F$ is Bivariate exponential against the alternative hypothesis

$H_{1}: F$ is BRNBU and not bivariate exponential.

Consider the bivariate exponential distribution introduced by [5], given by

$$
\bar{F}(x, y)=\exp \left(-\lambda_{1} x-\lambda_{2} y-\lambda_{12} \max (x, y)\right),
$$


ISSN: 2456-8686, 5(1), 2021:001-010

https://doi.org/10.26524/cm87

for all $x, y, \lambda_{1}, \lambda_{2}>0$ and $\lambda_{12} \geq 0$, where

$$
\begin{aligned}
\lambda_{1} & =\frac{\mu_{1}+\mu_{2}}{\mu_{12}}-\frac{1}{\mu_{2}} \\
\lambda_{2} & =\frac{\mu_{1}+\mu_{2}}{\mu_{12}}-\frac{1}{\mu_{1}} \\
\lambda_{12} & =\left(\frac{1}{\mu_{1}}+\frac{1}{\mu_{2}}\right) \frac{\mu_{12}-\mu_{1} \mu_{2}}{\mu_{12}} \\
\mu_{1} & =\int_{0}^{\infty} \bar{F}(x, 0) d x \\
\mu_{2} & =\int_{0}^{\infty} \bar{F}(0, y) d y \\
\text { and } \mu_{12} & =\int_{0}^{\infty} \int_{0}^{\infty} \bar{F}(x, y) d x d y .
\end{aligned}
$$

Define

$$
\delta_{E}=\sum_{i=0}^{r}\left(\begin{array}{l}
r \\
i
\end{array}\right) \frac{\mu_{r-i+2} \mu_{i+2}}{(r-i+1)(r-i+2)(i+1)(i+2)}-\frac{\mu \mu_{r+3}}{(r+2)(r+3)}
$$

Note that under $H_{0}, \quad \delta_{E}=0, \quad$ while under $H_{1}, \delta_{E}>0$. Let $\left(X_{1}, X_{2}\right),\left(X_{3}, X_{4}\right), \ldots,\left(X_{i-1}, X_{i}\right), \ldots\left(X_{n-1}, X_{n}\right)$ be a bivariate random sample from a distribution $F$.

Taking $r=1$ in equation (5), we get

$$
\delta_{E}=\frac{1}{6} \mu_{2} \mu_{3}-\frac{1}{12} \mu_{1} \mu_{4}
$$

The estimate $\widehat{\delta}_{E}$ of $\delta_{E}$ can be obtained as

$\widehat{\delta}_{E}=\frac{1}{n^{2}} \sum_{i=1}^{n} \sum_{j=1}^{n} \phi\left(X_{i}, X_{j}\right)$, where $\phi\left(X_{i}, X_{j}\right)=\frac{1}{6} X_{i}^{2} X_{j}^{3}-\frac{1}{12} X_{i} X_{j}^{4}$.

To make the test statistic scale invariant, let

$\widehat{\Delta}_{E}=\frac{\widehat{\delta}_{E}}{\mu^{r+4}}=\frac{1}{\mu^{r+4}}\left[\frac{1}{n^{2}} \sum_{i=1}^{n} \sum_{j=1}^{n}\left(\frac{1}{6} X_{i}^{2} X_{j}^{3}-\frac{1}{12} X_{i} X_{j}^{4}\right)\right]$

Then $\widehat{\Delta}_{E}$ is equivalent to the classical U-statistic, [4] and is given by $U_{n}=$ $\frac{1}{\left(\begin{array}{c}n \\ 4\end{array}\right)} \sum_{i<j \leq n} \phi\left(X_{i}, X_{j}\right)$.

The asymptotic normality of $\widehat{\Delta}_{E}$ is summarized in the following theorem. 
ISSN: 2456-8686, 5(1), 2021:001-010

https://doi.org/10.26524/cm87

Theorem 4.1 As $n \rightarrow \infty, \sqrt{n}\left(\widehat{\Delta}_{E}-\delta_{E}\right)$ is asymptotically normal with mean zero and variance $\sigma^{2}$, where

$$
\sigma^{2}=\operatorname{Var}\left\{\frac{1}{6} X_{i}^{2} \mu_{3}-\frac{1}{12} X_{i} \mu_{4}+\frac{1}{6} \mu_{2} X_{j}^{3}-\frac{1}{12} \mu X_{j}^{4}\right\}
$$

Here $\mu$ denotes the mean of $F$. Under $H_{0}$, the variance reduces to $\sigma^{2}=56$.

Proof: From the standard theory of U-statistic, [4], we have

$$
\sigma^{2}=\operatorname{Var}\left\{\varsigma\left(X_{i}, X_{j}\right)\right\}
$$

where

$$
\begin{aligned}
\varsigma\left(X_{i}, X_{j}\right) & =E\left[\phi\left(X_{i}, X_{j}\right) \mid X_{i}\right]+E\left[\phi\left(X_{i}, X_{j}\right) \mid X_{j}\right] \\
E\left[\phi\left(X_{i}, X_{j}\right) \mid X_{i}\right] & =\frac{1}{6} X_{i}^{2} \mu_{3}-\frac{1}{12} X_{i} \mu_{4} \\
E\left[\phi\left(X_{i}, X_{j}\right) \mid X_{j}\right] & =\frac{1}{6} \mu_{2} X_{j}^{3}-\frac{1}{12} \mu X_{j}^{4}
\end{aligned}
$$

and

$$
\varsigma\left(X_{i}, X_{j}\right)=\frac{1}{6} X_{i}^{2} \mu_{3}-\frac{1}{12} X_{i} \mu_{4}+\frac{1}{6} \mu_{2} X_{j}^{3}-\frac{1}{12} \mu X_{j}^{4}
$$

Thus $\sigma^{2}=\operatorname{Var}\left\{\frac{1}{6} X_{i}^{2} \mu_{3}-\frac{1}{12} X_{i} \mu_{4}+\frac{1}{6} \mu_{2} X_{j}^{3}-\frac{1}{12} \mu X_{j}^{4}\right\}$.

Under $H_{0}$,

$$
\varsigma_{0}\left(X_{i}, X_{j}\right)=X_{i}^{2}-2 X_{i}+\frac{1}{3} X_{j}^{3}-\frac{1}{12} X_{j}^{4}
$$

From the equation (6), it is clear that $E\left[\varsigma_{0}\left(X_{i}, X_{j}\right)\right]=0$ and

$\sigma^{2}=E\left[\left(\varsigma_{0}\left(X_{i}, X_{j}\right)\right)^{2}\right]=56$.

This completes the proof of the theorem.

Corollary 4.2 Under $H_{0}$, the limiting distribution of $U_{n}$ is normal with mean $\widehat{\Delta}_{E}$. The variance of $\sqrt{n}\left(U_{n}\right)$ is a function of $\lambda_{1}, \lambda_{2}$ and $\lambda_{12}$.

Proof: Since the variances of $\sqrt{n}\left(U_{n}\right)$ is very complicated under $H_{0}$ and since $U_{n}$ is a function of $\mathrm{U}$-statistic, jackknifing would not only reduce the bias, but also enable us to estimate the variance of $V\left(\sqrt{n} U_{n}\right)$. 
ISSN: 2456-8686, 5(1), 2021:001-010

https://doi.org/10.26524/cm87

The estimate of $V\left(\sqrt{n} U_{n}\right)$ is $\widehat{V}\left(\sqrt{n} U_{n}\right)=\frac{n}{n-1} \sum_{i=1}^{n}\left[U_{n, i}-U_{n}^{*}\right]^{2}$

where $U_{n, i}=U_{n-1}\left(X_{1}, \ldots, X_{i-1}, X_{i+1}, \ldots, X_{n}\right)$ and $U_{n}^{*}=\frac{1}{n} \sum_{i=1}^{n} U_{n, i}$

Using the results from $[7], \frac{\sqrt{n}\left(U_{n}\right)}{\left[\widehat{V}\left(\sqrt{n} U_{n}\right)\right]^{\frac{1}{2}}}$ is asymptotically standard normal.

Thus $\frac{\sqrt{n}\left(U_{n}\right)}{\left[\widehat{V}\left(\sqrt{n} U_{n}\right)\right]^{\frac{1}{2}}} \sim N(0,1)$.

This completes the proof of the corollary.

\section{Monte Carlo Simulations}

In this section the Monte Carlo null distribution critical points of $\widehat{\Delta}_{E}$ are simulated based on 50000 generated samples of size $n=5(5) 100$. Table 1 gives the upper percentile points of statistic $\widehat{\Delta}_{E}$ for different confidence levels $90 \%, 95 \%, 98 \%$ and $99 \%$.

Table 1. Critical Values of the statistic $\widehat{\Delta}_{E}$

\begin{tabular}{|c|llll|}
\hline $\mathrm{n}$ & $90 \%$ & $95 \%$ & $98 \%$ & $99 \%$ \\
\hline 5 & 0.027 & 0.062 & 0.157 & 0.290 \\
10 & 0.061 & 0.128 & 0.288 & 0.487 \\
15 & 0.090 & 0.178 & 0.388 & 0.636 \\
20 & 0.117 & 0.222 & 0.465 & 0.737 \\
25 & 0.148 & 0.270 & 0.546 & 0.877 \\
\hline 30 & 0.172 & 0.311 & 0.623 & 0.954 \\
35 & 0.204 & 0.363 & 0.687 & 1.028 \\
40 & 0.224 & 0.395 & 0.748 & 1.142 \\
45 & 0.252 & 0.438 & 0.828 & 1.271 \\
50 & 0.272 & 0.469 & 0.883 & 1.354 \\
\hline
\end{tabular}


ISSN: 2456-8686, 5(1), 2021:001-010

https://doi.org/10.26524/cm87

\begin{tabular}{|c|cccc|}
\hline 55 & 0.290 & 0.491 & 0.921 & 1.432 \\
60 & 0.313 & 0.542 & 1.008 & 1.510 \\
65 & 0.328 & 0.552 & 1.030 & 1.581 \\
70 & 0.352 & 0.598 & 1.071 & 1.639 \\
75 & 0.359 & 0.603 & 1.114 & 1.678 \\
\hline 80 & 0.389 & 0.651 & 1.212 & 1.780 \\
85 & 0.403 & 0.659 & 1.180 & 1.837 \\
90 & 0.429 & 0.702 & 1.242 & 1.869 \\
95 & 0.439 & 0.736 & 1.306 & 1.955 \\
100 & 0.468 & 0.760 & 1.310 & 2.026 \\
\hline
\end{tabular}

Figure 1: Relation between critical values, sample size and confidence levels

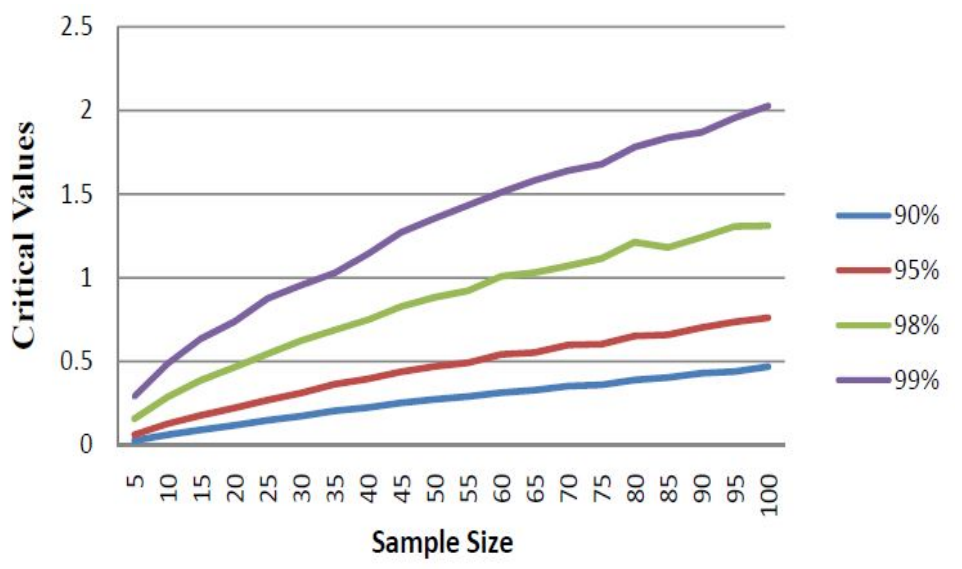

It is clear from Table 1 and Figure 1, that the critical values increase as the confidence level increases and almost increases as the sample size increase.

\section{Application to Real Life Data}

Here, we present a real life example to illustrate the use of our test statistics $\widehat{\Delta_{E}}$. We consider the example given by [2] which is a list of paired first failure time (in hours) of the transmission and the transmission pump on 15 Caterpillar tractors. We use our test to detect whether these failure times follow a bivariate exponential 
ISSN: 2456-8686, 5(1), 2021:001-010

https://doi.org/10.26524/cm87

distribution.

Table 2. First failure times of transmission $\left(X_{i}\right)$ and transmission pump $\left(X_{j}\right)$ on D9G-66A Caterpillar Tractors.

\begin{tabular}{|c|c|c|c|}
\hline Tractor Number & $X_{i}$ & $X_{j}$ & $\min \left(X_{i}, X_{j}\right)$ \\
\hline 1 & 1641 & 850 & 850 \\
2 & 5556 & 1607 & 1607 \\
3 & 5421 & 2225 & 2225 \\
4 & 3168 & 3223 & 3168 \\
5 & 1534 & 3379 & 1534 \\
\hline 6 & 6367 & 3832 & 3832 \\
7 & 9460 & 3871 & 3871 \\
8 & 6679 & 4142 & 4142 \\
9 & 6142 & 4300 & 4300 \\
10 & 5995 & 4789 & 4789 \\
\hline 11 & 3953 & 6310 & 3953 \\
12 & 6922 & 6310 & 6310 \\
15 & 4210 & 6378 & 4210 \\
\hline 12 & 5161 & 6449 & 5161 \\
\hline 12 & 6949 & 4732 \\
\hline
\end{tabular}

Using the data in Table 2, we obtain, the value of the test statistic $\widehat{\Delta}_{E}=0.0000151947$. Thus $\frac{\sqrt{n}\left(U_{n}\right)}{\left[\widehat{V}\left(\sqrt{n} U_{n}\right)\right]^{\frac{1}{2}}}=8.263809664 \times 10^{9}$.

Hence, we reject $H_{0}$ and conclude in favour of BRNBU.

Then we accept $H_{1}$ which shows that the data set has Bivariate Renewal New Better than Used property, but not Bivariate exponential. 
ISSN: 2456-8686, 5(1), 2021:001-010

https://doi.org/10.26524/cm87

\section{Conclusion}

The Bivariate Renewal New Better than Used Ageing Class of life-time distribution is considered. The moment inequality is derived. A new test statistic for testing Bivariate Exponentiality against Bivariate Renewal New Better than Used Ageing class of life-time distributions based on the moment inequality is proposed. Using Monte Carlo Method, critical values of the proposed test are calculated for $n=5(5) 100$ and tabulated. Finally, application to real-life data is given.

\section{References}

[1] Ahmad I.A. Moments inequalities of aging families of distributions with hypothesis testing applications, Journal of Statistical Planning and Inference, 92, 121-132 (2001).

[2] Barlow R.E and Proschan F. Techniques for analyzing Multivariate data, Theory and Applications of Reliability, Academic Press, New York, 1977.

[3] Hoeffding W.A. A Class of Statistics with Asymptotically Normal Distribution, Annals of Mathematical Statistics, 2, 293-325 (1948).

[4] Lee A .J.U-Statistic. Marcel Dekker, New York , 1989.

[5] Marshall A. W. and Olkin I. A Multivariate Exponential Distribution, Journal of the American Statistical Association, 62, 30-44 (1967).

[6] Sathiyaraj A and Rizwan U.New Moment Inequalities for Some Bivariate Ageing Classes, International Journal of Research Analytical Reviews, 6(1), 719-726 (2019).

[7] Sen P.K. Some Invariance Principles Relating to Jackknifing and their Role in Sequential Analysis, Annals of Statistics, 5, 316-329 (1977).

[8] Suresh R. P. A simple inequality of moments in some classes of bivariate ageing disrtibutions, Journal of Indian Statistical Association, 39, 131-136 (2001).

[9] Syed Tahir Hussainy, Sathiyaraj A and Rizwan U. Moment Inequalities for Some Bivariate Ageing Classes, International Journal of Current Advanced Research, $7(1), 48-57$ (2018).

[10] Touseef Ahmed. A and Rizwan. U. Testing BHNBUE Class of Life-Time Distribution Based on Moment Inequality, Advances in Mathematics: Scientific Journal, 9(8), 6261-6268 (2020). 